\title{
1.3.3 Consequences of the Inclination of the Zodiacal Cloud
} on the Ecliptic

\author{
R. Robley \\ U.E.R. Observatoires du Pic du Midi et de Toulouse/France
}

Assuming that the decrease in the density of the interplanetary dust follows an exponential distribution both in the transverse and radial direction, we can write $n=n_{0} \operatorname{Exp}(-(h / H)-(r-1 / R))$, where $h$ is the distance from the ecliptic plane and $r$ the heliocentric distance both expressed in astronomical units (a.u.); then we show that the modulation of the radiance $B(90,0)$ of the zodiacal light observed at the ecliptic pole defines the parameter $\mathrm{H}$ as a function of the inclination angle $B$ between the zodiacal cloud and the ecliptic plane; moreover, the experimental value of the ratio $B(90,0) / B(90,90)$ defines the parameter $R$. It can be deduced that the flatness of the zodiacal cloud, expressed by $R / H$, is $<5$ and that the plane of symmetry of the zodiacal cloud is very close to that of the invariant plane of the solar system $\left(\beta<2^{\circ}\right)$.

Assuming that the composition of the interplanetary medium is mainly $\mathrm{Fe}$ and $\mathrm{SiO}_{2}$ grains (not including lee for which the estimated life time is too short), we show that only the dust grains with a size distribution favorising the submicronic grains give a ratio $\mathrm{B}(90,0) /$ $\mathrm{B}(90$, 90) close to the observed value.

The degrees of polarization computed for some mixtures of $\mathrm{Fe}$ and $\mathrm{SiO}_{2}$ do not agree very well with the observations; using an inverse power law for the size distribution of the radii, say $n(a) \sim a^{-p}$, it seems that the law would be better with $p=3$ or 3.5 than with $p=4$.

The full paper will be published in Astron. and Astrophys. 